\title{
Perspectives for the VITO beam line at ISOLDE, CERN
}

\author{
R. F. Garcia Ruiz ${ }^{1, a}$, M.L. Bissell ${ }^{1}$, A. Gottberg ${ }^{2}$, M. Stachura ${ }^{2,3}$, L. Hemmingsen ${ }^{3}$, G. Neyens ${ }^{1}$, N. Severijns ${ }^{1}$, and \\ the VITO Collaboration \\ ${ }^{1}$ Instituut voor Kern- en Stralingsfysica, KU Leuven, Celestijnenlaan 200 D, 3001 Leuven, Belgium \\ ${ }^{2}$ CERN, 1211 Geneva 23, Switzerland \\ ${ }^{3}$ Kemisk Institut, Københavns Universitet Universitetsparken 5, 2000 København, Denmark
}

\begin{abstract}
By using polarized ion beams in combination with the $\beta$-NMR technique, the Versatile Ion-polarized Techniques On-line (VITO) experiment at ISOLDE, CERN links together expertise from different fields in an unique experimental setup. An overview of the experimental techniques and a general description of the newly designed beam line are presented. Potential uses in multidisciplinary research and perspectives for future experiments are discussed.
\end{abstract}

\section{Introduction}

The principle of $\beta$-asymmetry detection on polarized nuclei has been established for more than half a century [1]. By implanting polarized nuclei in a medium and subsequent detection of its $\beta$-asymmetry, valuable information from either, the nucleus or the implantation medium can be inferred. With the recent developments of radioactive beam line facilities [2], the use of polarized nuclei has opened new opportunities to elucidate unexplored physical phenomena in nuclear structure [3-5], weak interactions studies [6-9] and material sciences [10].

At ISOLDE, CERN [11], polarized nuclei can be obtained by applying one of the commonly used techniques: laserinduced nuclear orientation [12], low temperature nuclear orientation [13], or the tilted foils method in combination with the post-accelerated beams from REX-ISOLDE [14]. Previous experimental programs were mainly focused on nuclear structure [4] and weak interaction studies [15-17]. Only recently, this technique has been applied successfully in the field of chemistry and bio-chemistry [18]. These latest results motivated the foundation of a new dedicated beam line for laser-induced nuclear orientation, currently known as the Versatile Ion-polarized Techniques On-line (VITO) experiment at ISOLDE. Here, we describe the general aspects of the new beam line. The basic principles of the experimental techniques are revised to illustrate its applications in different research fields. Finally, some of the planed experimental programs are briefly described.

\section{VITO Beam line}

On-line laser-induced nuclear orientation in combination with $\beta$-NMR was introduced for the first time at ISOLDE

\footnotetext{
${ }^{\mathrm{a}} \mathrm{e}$-mail: RonaldFernando.GarciaRuiz@fys.kuleuven.be
}

[12]. At present there is only one dedicated beam line for laser-induced nuclear orientation, namely at TRIUMF, Canada [19]. Besides being a unique alternative for users across Europe, VITO will include unprecedented features, such the $\beta$-NMR chamber for experiments on liquid samples, and a UHV $\beta$-NMR set-up for studies of surfaces and interfaces. The new beam line was proposed as a modification of the former UHV beam line, previously hosting the Apparatus for Surface Physics and Interfaces at CERN (ASPIC) [20]. Two major upgrades are currently under construction; i) an optical pumping zone for laser-induced nuclear orientation, and ii) three end stations for multiple users of polarized beams. The previous ASPIC apparatus is preserved as one of the end stations. A second station will include the recently developed bio $\beta$-NMR chamber [21] for experiments in biophysics and chemistry. A third open station, on a straight line, will serve for monitoring the degree of polarization, or for hosting a movable experiment.

A schematic drawing of the new beam line is shown in Figure 1. The mass separated ion beams enter the beam line, where they overlap with a laser beam in a collinear geometry. The ion velocity can be varied by changing the electric potential in the interaction region. Using the Doppler effect, the resonance laser frequency can be scanned across different hyperfine transitions. By using circularly polarized laser light, along with a weak magnetic field $(\sim 0.4$ $\mathrm{mT})$ in the direction of the beam, the quantization axis of the atomic (and nuclear) polarization is defined. For some species, the optical pumping process is desired on the atomic states rather than the ionic states. Therefore, a charge exchange cell filled with a gas of alkali atoms can be used to neutralize the incoming ion beam. Once the atoms interact with the laser beam, and nuclear polarization is achieved, the polarized atoms are re-ionized to be redirected towards the different end stations. 


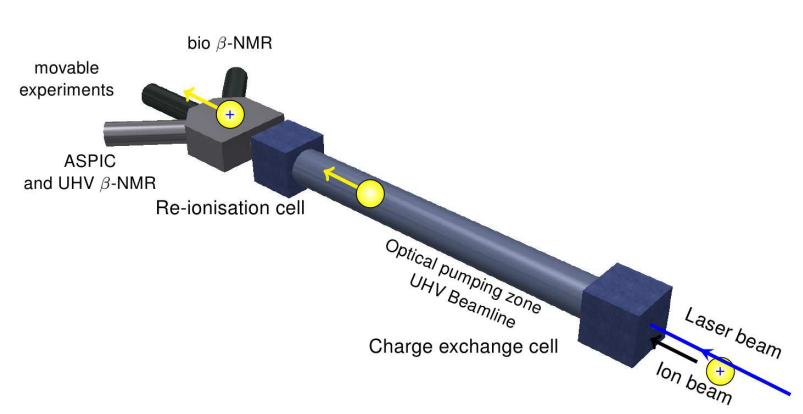

Figure 1. Schematic drawing of the VITO beam line.

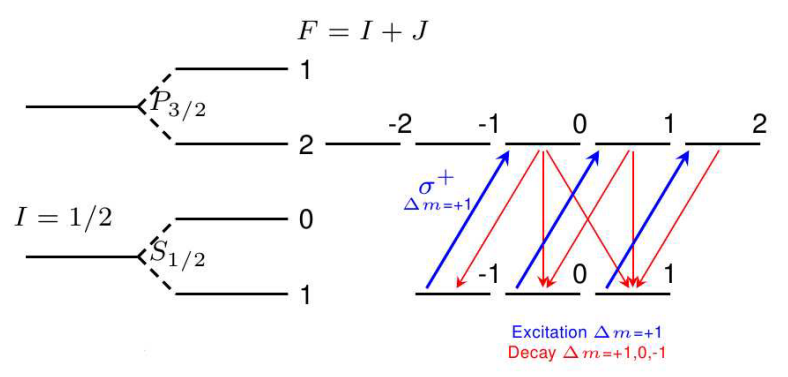

Figure 2. Scheme of optical pumping for a transition $F=1 \rightarrow$ $F=2$ with circularly polarized light $\sigma^{+}$.

\subsection{Laser-induced nuclear orientation}

When atoms or ions interact with circularly polarized laser light $\sigma^{+}\left(\sigma^{-}\right)$, the absorption process is only allowed following the selection rules $\Delta m_{F}=+1\left(\Delta m_{F}=-1\right)$. Figure 2 shows an example of a hyperfine structure scheme for an atomic transition $S_{1 / 2} \rightarrow P_{3 / 2}$ for a nucleus with spin $I=1 / 2$. Incident polarized light $\sigma^{+}$stimulates absorption among the magnetic sub-states of the different hyperfine transitions, e.g, $F=1 \rightarrow F=2$. Spontaneous decay can occur following $\Delta m_{F}=-1,0,+1$, but the circularly polarized light re-pumps these states with $\Delta m=+1$. As a consequence the total population is transferred to magnetic sub-states with higher magnitude of angular momentum. As the laser-atom interaction is a well known process, the polarization can be predicted with relatively good accuracy $[22,23]$. A major advantage of this method is the high degree of nuclear polarization that can be achieved, typically between $10 \%$ and $80 \%$, depending on the polarization scheme.

\subsection{The $\beta$-NMR technique}

Polarized nuclei can be implanted in a host crystal or a liquid medium for NMR experiments (see Figure 3-a). At the VITO beam line, the ions are electrostatically deflected with $90^{\circ}$ respect to the beam direction, while the initial direction of the polarization axis is preserved. Once the nuclei are implanted, the electron and nuclear spin are decoupled and the nuclear polarization is maintained thanks to a strong magnetic field that is applied along the polarization

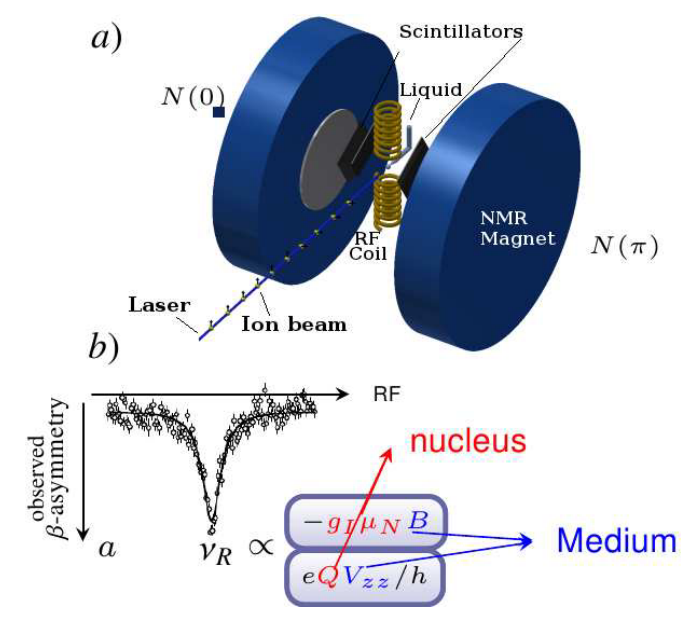

Figure 3. a) Sketch of the experimental setup for $\beta$-NMR experiments in liquids. $b$ ) Example of a $\beta$-NMR signal (see text for more details).

direction. $\beta$ particles are emitted following the distribution [24]

$$
W(\theta)=1+\frac{v}{c} A_{\beta} P \cos \theta
$$

where $P$ is the nuclear polarization, and $A_{\beta}$ is the $\beta$ asymmetry parameter, expressed for allowed transitions as

$$
A_{\beta}= \begin{cases} \pm 1 & \text { for } I_{f}=I_{i}-1 \\ \mp \frac{I_{i}}{I_{i}+1} & \text { for } I_{f}=I_{i} \\ 1+\rho^{2} & \text { for } I_{f}=I_{i}+1 .\end{cases}
$$

Where $I_{i}$ and $I_{f}$ are the initial and final nuclear spin involved in the $\beta$-decay process, respectively. $\rho$ stands for the Gamow-Teller to Fermi mixing ratio, and the upper(lower) sign is for positron(electron) emission.

The experimental $\beta$-asymmetry, $a$, is obtained as the normalized difference between the $\beta$ particles detected at $0^{\circ}$, $N(0)$, and the $\beta$ particles detected at $180^{\circ}, N(\pi)$, given by

$$
a=\frac{N(0)-N(\pi)}{N(0)+N(\pi)} \equiv \frac{v}{c} A_{\beta} P .
$$

The splitting into nuclear magnetic sub-states is originating from the interaction between the fields induced by the host material with the nuclear magnetic moment, $\mu$, and the nuclear quadrupole moment, $Q$. Depending on the implantation medium an electric field gradient, $V_{z z}$, can be produced at the site of the implanted nuclei. Together with the Zeeman splitting induced by the applied static field and the field of the host, $B$, a splitting into magnetic sub-levels is created. If an external radio-frequency (RF) magnetic field is applied, transitions among these magnetic sub-levels can be induced, giving rise to a loss of the nuclear spin polarization. A resonant destruction of the nuclear spin polarization can be directly measured by a change of the experimental $\beta$-asymmetry as a function of the applied RF-frequency. An example of a $\beta$-NMR signal is shown in Figure 3-b. The resonant frequency, $v_{R}$, is a 
direct measurement of the energy splitting induced by the magnetic and the quadrupole hyperfine interactions and allows us to deduce the Larmor frequency, $v_{L}=-g_{I} \mu_{N} B$, or the quadrupole frequency, $e Q V_{z z} / h$.

By measuring the Larmor frequency, $v_{L}$, the nuclear magnetic moment can be obtained, provided that $B$ is known [25]. However, if the nuclear moments are measured independently, the nucleus can be used as a local electromagnetic probe to obtain $B$ and $V_{z z}$ inside the implantation medium [26]. Additional information on the electromagnetic properties of the medium can be obtained by measuring the nuclear polarization as a function of time (spin relaxation [27]) during and after the implantation. On the other hand, the $\beta$-asymmetry parameter can be measured (eq. 2) to extract the nuclear spins and parities of ground and excited nuclear states. In addition, when the nuclear spins are established, $A_{\beta}$ can be used to obtain $\rho^{2}$ [28], which can serve as test of the unitary of the Kobayashi-Maskawa (CKM) quark mixing matrix, via $V_{u d}^{2} \propto\left(1+\frac{f_{A}}{f_{V}} \rho^{2}\right)^{-1}[29]$. An extension of the experimental methods, plans and perspectives for futures experiments in the VITO beam line are presented in the following sections.

\section{First results and opportunities for future experiments}

\subsection{Biophysics}

With the recently developed bio $\beta$-NMR chamber [21], the $\beta$-NMR technique was for the first time successfully applied to record an NMR spectrum in a solution [18], opening new possibilities for applications in general chemistry and biochemistry. A photograph of the core of the chamber is shown in Figure 4. During the experimental campaign, the chamber was tested at the end of the COLLAPS beam line, but later will be placed as one of the end stations of the VITO Beam line. The experimental setup is illustrated in Figure 3-a. Polarized ${ }^{31} \mathrm{Mg}$ ions were implanted directly into an ionic liquid. By using a RF coil around the implantation point, the external RF frequency was scanned to locate the resonance NMR signals. A MgO crystal was used as a reference sample. Details of the experimental results can be found in Ref. [18].

Two main experimental challenges are faced when liquid samples are used, i) interface between extreme vacuum environments, ranging from $10^{-6}$ mbar up to few mbar, ii) preservation of the nuclear polarization through the vacuum /liquid interface. An ionic liquid (EMIM-OAc) of very low vapor pressure was used during the experiment to explore systematically the behaviour of ion transmission and nuclear polarization at different pressures. Currently, improvements of the design are ongoing to allow the possibility of using water as solvent, to extend applications to general chemistry and biochemistry. When comparing $\beta$-NMR with conventional NMR, both experimental techniques provide similar information: local structure and dynamics at the probe site through detection of chemical shifts and spin relaxation times. The vast difference

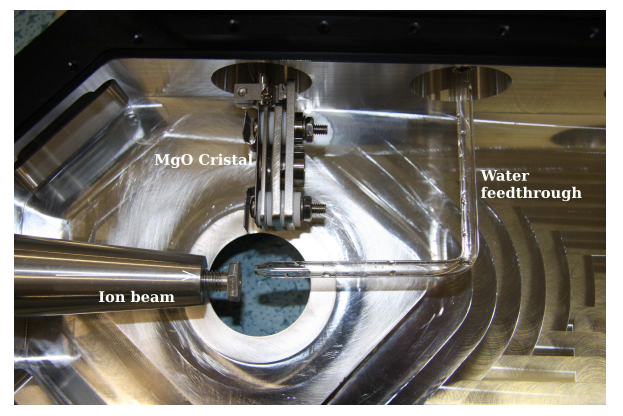

Figure 4. Photograph of the core of the chamber used for $\beta$-NMR spectroscopy on liquid samples.

lies in the sensitivity. While $\sim 10^{16}$ atoms are normally required in conventional NMR, only $\sim 10^{7}$ atoms are needed for $\beta$-NMR studies. In addition, the $\beta$-NMR can be used for elements which are otherwise difficult to observe spectroscopically in their closed electronic shell oxidation state such as $\mathrm{Mg}^{2+}, \mathrm{Ca}^{2+}, \mathrm{Cu}^{+}$and possibly $\mathrm{Zn}^{2+}$. These metal ions are ubiquitous in biology with functions in for example electron transport, redox reactions, enzyme catalysis, regulations of gene expression and many other reaction pathways, as well as nucleic acid biochemistry [30]. Conversely, the concentrations of most of these metal ions are maintained at highly regulated levels in cells, and the structure and functions of transmembrane proteins selectively pumping metal ions into or out of cells is a current hot topic [31-33]. Thus, there is a considerable need for spectroscopic methods that may characterize the metal site coordination geometries of these metal ions in biological systems. A letter of intent to explore the technique on these ions [34], and a full proposal to study $\mathrm{Mg}$ [35] and $\mathrm{Cu}$ [36] have already been accepted at ISOLDE. Experiments are expected to start in 2015 .

\subsection{Material sciences}

Applications of $\beta$-NMR spectroscopy for studies on material science are rather well-known [26]. As it was previously mentioned, a nucleus with known electromagnetic properties can be used as an electromagnetic probe to extract the electric and/or magnetic properties of a given implantation or binding state $[10,37]$. By controlling the implantation energy, material properties can be explored at nano-metric depths [38, 39]. Magnetic and electric properties can be separated by selecting isotopes with different nuclear spin, e.g, a nucleus with spin $I=1 / 2$, and therefore no quadrupole moment, will exhibit a purely magnetic interaction with the host lattice. Furthermore, phenomena at different time scales can be studied by choosing a nucleus with an appropriate lifetime, commonly from few tens of milliseconds up to few seconds.

The previous ASPIC apparatus will be preserved as one of the end stations of the VITO beam line, providing continuity to foregoing experimental programs in the field of surface physics [40-43]. At ASPIC, state-of-theart techniques are used for in situ sample preparation and characterization, to study surfaces, interfaces and layers in 
a UHV environment ( 10 $0^{-10}$ mbar). Radioactive nuclei are collected from ISOLDE and deposited with thermal energy on the top of the first atomic layer via soft-landing. Afterwards, electromagnetic properties of the sample can be measured using Perturbed Angular Correlation (PAC) spectroscopy [44]. A further development will adapt a chamber for $\beta$-NMR studies inside of ASPIC. Combinations of PAC and NMR have been applied in the past as complementary methods (see for example [45] and [46] for nuclear physics and biophysics applications, respectively). Albeit PAC and $\beta$-NMR provide similar information, both methods are sensitive to very different time scales. Dynamic properties can be studied in the range of 0.1 to 100 ns using PAC spectroscopy, while in $\beta$-NMR the time scale ranges from few hundreds of milliseconds to few seconds.

\subsection{Nuclear structure}

Polarization by optical pumping and subsequent detection of the $\beta$-asymmetry has been extensively used at ISOLDE to obtain nuclear structure information. Nuclear ground states properties as magnetic and quadrupole moments, have been previously measured on different isotopes at the COLLAPS beam line $[4,5,23,47,48]$. As it is not the purpose of the VITO experiment, the discussion will only be extended on alternative experiments that can take advantage of particular features of the new beam line, e.g., $\beta$-delayed spectroscopy of laser-polarized beams [49].

In addition to the ground state properties, spin and parity of excited states in the $\beta$-decay can be obtained by using $\beta$ delayed spectroscopy of polarized beams [50]. A scheme of a nuclear decay is shown in Figure 5. Excited states populated in the $\beta$-transition can decay either by particle emission $(\alpha, \beta, n)$, or $\gamma$-decay. The value $A_{\beta} P$ (Eq. 3$)$ for decay to a specific excited state can be obtained by measuring the $\beta$-asymmetry in coincidence with the delayed $\gamma$-rays (or particles). As the polarization, $P$, is the same for all transitions, it can be obtained, e.g., by measuring the asymmetry of a pure GT decay branch (for which $A_{\beta}$ is known to be 1). As the value $A_{\beta}$ depends on the spins of the initial, $I_{i}$, and final state, $I_{f}$, involved in the transition (Eq. 2). Measuring the $\beta$-asymmetry of a particular transition (and thus its $A_{\beta}$ ) allows unambiguous spin and parity assignments in the daughter nucleus.

In addition to the $\beta$-counters normally employed in $\beta$ NMR, this technique requires supplementary detector arrays. $\gamma$-detectors can be used to tag low lying excited states. An experimental campaign for including such kind of experiments at the VITO beam line is ongoing [34].

\subsection{Fundamental weak interaction physics}

Testing the unitary of the Cabibbo-Kobayashi-Maskawa (CKM) quark mixing matrix, constitutes an important test to our understanding of the fundamental physics. A deviation from unity indicates the existence of new physics not included in the standard model [28].

The highest precision value of $V_{u d}$, has been obtained from

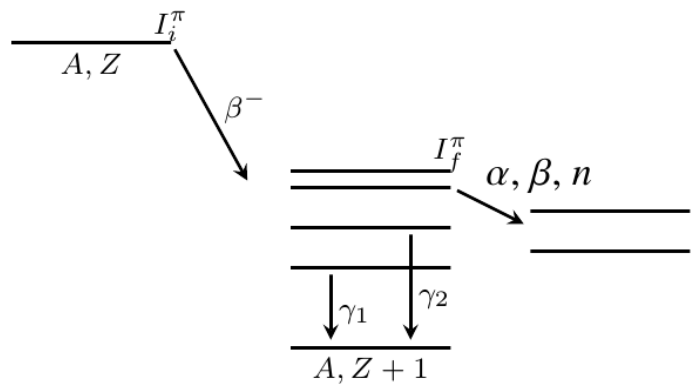

Figure 5. Nuclear decay scheme for $\beta$-delayed spectroscopy.

$0^{+} \rightarrow 0^{+}$super allowed $\beta$-decay, $V_{u d}=0.97425(22)$ [51]. An alternative method to obtain an independent high precision value of $V_{u d}$ was suggested from $T=1 / 2$ nuclear mirror transitions [52]. As it was noticed (Eq. 2), for $\beta$ decay transitions with $I_{i}=I_{f}$, the $\beta$-asymmetry depends on the Gamow-Teller to Fermi mixing ratio, $\rho$. If the corrected $F t$-value is known for the same transition, a measurement of $\rho$ can be used to evaluate the matrix element $V_{u d}$, via [52]

$$
V_{u d}^{2}=\frac{2 \pi^{3} \hbar \ln 2}{m_{e} c^{2}} \frac{1}{F t G_{F}^{2}\left(1+\Delta_{R}^{V}\right)}\left(1+\frac{f_{A}}{f_{V}} \rho^{2}\right)^{-1},
$$

with $m_{e}$ the electron mass, $c$ speed of light, and $G_{F}$ the Fermi constant [53]. $f_{V}$ and $f_{A}$ are the statistical rate functions for the vector and axial-vector part of the interaction. A radiative correction, $\Delta_{R}^{V}$, should be applied for the respective transition [54]. In mirror transitions, $V_{u d}$ is highly sensitive to the value of $\rho$. A special example is found in ${ }^{35} \mathrm{Ar}$ [55], where it was shown that a relative precision of $0.5 \%$ on the $\beta$-asymmetry parameter, allows for a determination of $V_{u d}$ with a relative precision of $\sim 0.07 \%$. This is an impressive result, considering that in a single measurement, the obtained uncertainty is only three times less that the uncertainty of the average value extracted from the entire set of high precision measurements from super allowed $0^{+} \rightarrow 0^{+}$transitions.

The value $A_{\beta} P$ can be directly obtained from the experimental $\beta$-asymmetry (Eq. 2). Nevertheless, a precise determination of $\rho$ relies on the precise measurement of both $A_{\beta}$ and $P$. High precision measurements of such quantities impose experimental challenges on the development of laser polarization schemes, choice of suitable implantation crystals, and methods to determine the total polarization efficiency. A current project to measure the $\beta$-asymmetry parameter of ${ }^{35} \mathrm{Ar}$ has been proposed at the VITO beam line [29].

\section{Conclusions}

Spin-polarized beams of radioactive species represent a powerful tool to explore a large variety of physical phenomena. Versatility has been the key factor of the newly designed VITO beam line at ISOLDE. Using laserinduced nuclear orientation in combination with $\beta$-NMR opens the possibility of performing experiments in a broad 
spectrum of disciplines. Furthermore, a novel experimental setup was developed to apply $\beta$-NMR directly in liquid samples. This setup will allow the study of ions that are difficult to study by other spectroscopic techniques.

Thanks to the first successful results and the joint effort among experts from different fields at ISOLDE, a comprehensive experimental program is being prepared to benefit scientific research in biophysics, material sciences, nuclear structure and weak interaction physics.

\section{Acknowledgments}

This work was supported by the IAP-project P7/12, the FWO-Vlaanderen, the NSF grant PHY-1068217, BMBF(05 P12 RDCIC), the Danish Center for Scientific Computing for resources, and the Danish Council for Independent Research and Natural Sciences.

\section{References}

[1] C.S. Wu et al, Phys. Rev. 105, 1413 (1957)

[2] Y. Blumenfeld et al, Phys. Scr. T152, 014023 (2013)

[3] W. Geithner et al, Phys. Rev. Lett. 83, 3792 (1999)

[4] G. Neyens et al, Phys. Rev. Lett. 94, 022501 (2005)

[5] R. Neugart et al, Phys. Rev. Lett. 101, 132502 (2008)

[6] G. Soti et al, Phys. Rev. C 90, 035502 (2014)

[7] A. Etile et al, EPJ Web Conf. 66, 02034 (2014)

[8] J.R.A. Pitcairn et al, Phys. Rev. C 79, 015501 (2009)

[9] D. Melconian et al, Phys. Lett. B 649, 370 (2007)

[10] Z. Salman et al, Phys. Rev. Lett. 109, 257207 (2012)

[11] A. Herlert et al, Nucl. Phys. News 20, 5 (2010)

[12] E. Arnold et al, Phys. Lett. B 197, 311 (1987)

[13] J. Wouters et al, Hyp. Int. 59, 59 (1990)

[14] H. Tornqvist et al, Nucl. Inst. Meth. B 317, 685 (2013)

[15] N. Severijns et al, Phys. Rev. Lett. 63, 10 (1989)

[16] F. Wauters et al, Phys. Rev. C 82, 055502 (2010)

[17] J.R. Stone et al, Phys. Rev. C 76, 025502 (2007)

[18] A. Gottberg et al, Accepted in Chem. Phys. Chem. (2014)

[19] K.H. Chow et al, Physica B 340, 1151 (2003)

[20] H.H. Bertschat et al, Hyp. Int. 129, 475 (2000)

[21] A. Gottberg et al, In preparation (2014)

[22] M. Keim et al, Eur. Phys. J. A 8, 31 (2000)

[23] M. Kowalska et al, Phys. Rev. C 77, 034307 (2008)

[24] N. Stone and H. Postma, Low-temperature Nuclear Orientation (Elsevier, Amsterdam , 1986)
[25] G. Neyens et al, Rep. Prog. Phys. 66, 633 (2003)

[26] B. Ittermann et al, Hyp. Int. 79, 591 (1993)

[27] Z. Salman et al, Phys. Rev. B 70, 104404 (2004)

[28] N. Severijns et al, Rev. Mod. Phys. 78, 991 (2006)

[29] Ph. Velten et al, CERN-INTC-2014-062 / INTC-P426 (2014)

[30] I. Bertini et al, Biological inorganic chemistry structure reactions (University Science Books, 2007)

[31] M. Lu and D. Fu, Science 317, 5845 (2007)

[32] M. Hattori et al, Nature 448, 1072 (2007)

[33] P. Gourdon et al, Nature 475, 59 (2011)

[34] M. Deicher et al, CERN-INTC-2013-013 / INTC-O017 (2013)

[35] M. Stachura et al, CERN-INTC-2013-033 / INTC-P392 (2013)

[36] M. Stachura et al, CERN-INTC-2013-034 / INTC-P393 (2013)

[37] Z. Salman et al, Nano Lett. 7, 1551 (2007)

[38] G.D. Morris et al, Phys. Rev. Lett. 93, 157601 (2004)

[39] Z. Salman et al, Phys. Rev. Lett. 98, 167001 (2007)

[40] H.H Bertschat et al, Phys. Rev. Lett. 78, 342 (1997)

[41] H.H Bertschat et al, Phys. Rev. Lett. 80, 2721 (1998)

[42] W. Weber et al, Phys. Rev. B 64, 081404 (2001)

[43] H. Granzer et al, Phys. Rev. Lett. 77, 4261 (1996)

[44] H. Frauenfelder and R.M. Steffen, Alpha-, Beta- and Gamma-Ray Spectroscopy (Siegbahn, North-Holland, 1968)

[45] N. Brauer et al, Z. Physik 244, 375 (1971)

[46] L. Hemmingsen et al, J. Am. Chem. Soc. 123, 10329 (2001)

[47] D. Yordanov et al, Phys. Rev. Lett. 99, 212501 (2007)

[48] D. Yordanov et al, Phys. Rev. Lett. 108, 042504 (2012)

[49] D. Yordanov et al, CERN-INTC-2010-021 / INTC-I090 (2010)

[50] H. Miyatake et al, Phys. Rev. C 67, 014306 (2003)

[51] I. S. Towner and J. C. Hardy et al, Rep. Prog. Phys. 73, 046301 (2010)

[52] O. Naviliat-Cuncic and N. Severijns et al, Phys. Rev. Lett. 102, 142302 (2009)

[53] C. Amsler et al, Phys. Lett. B 667, 1 (2008)

[54] W.J. Marciano et al, Phys. Rev. Lett. 96, 032002 (2006)

[55] N. Severijns et al, Physica Scripta T152, 014018 (2013) 
